\title{
MORFETIK, UN DICTIONNAIRE MORPHOLOGIQUE : ILLUSTRATION AVEC LE LEXIQUE DE LA PERCEPTION
}

\author{
Aude GREZKA \\ Université Paris 13, Sorbonne Paris Cité \& CNRS (France) \\ UMR 7187 Laboratoire Lexiques Dictionnaires Informatique
}

\begin{abstract}
En): Presently, numerous works concern the reflection and the conception of appropriate methodologies for automatic language processing. Their objectives are to create a linguistic automatable resource which will be used on IT applications (assistant to writing, to translation, etc.). In this article, we present the morphological resource Morfetik, a morphological dictionary of the simple words of French. At first, we return to the difference between a computer dictionary and computerized dictionary. Secondly, we approach the structuring of Morfetik by illustrating it with the lexicon of the perception.
\end{abstract}

Keywords (En): morphological dictionary ; flexion ; lemma ; lexicon ; perception

Mots-clés (Fr) : dictionnaire morphologique ; flexion ; lemme ; lexique ; perception

\section{Introduction}

De nombreux travaux portent actuellement sur la réflexion et la conception de méthodologies appropriées au traitement automatique des langues. Il s'agit de modéliser une certaine partie de la connaissance linguistique afin de la rendre exploitable par la machine, de créer de la ressource linguistique qui sera employée dans des applications informatiques (aide à la rédaction, à la traduction, etc.). Dans ce travail, nous présentons la ressource morphologique Morfetik, un dictionnaire morphologique des mots simples du français. Dans un premier temps, nous revenons sur la différence entre dictionnaire informatique et dictionnaire informatisé. Dans un deuxième temps, nous abordons la structuration de Morfetik en l'illustrant avec le lexique de la perception.

\section{Problématique et axes de recherche}

Le traitement automatique des langues nécessite une identification précise des formes, une conceptualisation des phénomènes linguistiques. Cela suppose un recensement lexical complet et un degré suffisant de couverture, que peut apporter un « dictionnaire électronique » construit à cet effet.

Il est important de revenir sur la différence entre deux types de dictionnaires qui utilisent l'informatique : dictionnaire informatisé (ou numérique) et dictionnaire électronique (COURTOIS et SILBERZTEIN, 1990 ; GROSS, 2005).

Le dictionnaire informatisé représente la version électronique du dictionnairepapier et a l'avantage que la recherche de l'information y est infiniment plus rapide puisqu'il y a une véritable navigation dans le texte. Mais les informations que l'on est en mesure de mettre en évidence ne sont pas de nature différente de celles que l'on trouve dans un dictionnaire-papier. Ce type de dictionnaire a une norme de codage inhérente au phénomène de rétroconversion.

À ce dictionnaire informatisé, on opposera le dictionnaire électronique, qui est conçu dans un but de traitement automatique (reconnaissance ou génération). Toutes 
les informations y sont explicites et reproductibles. Puisqu'il a pour objectif le traitement automatique, il ne s'appuie pas sur des rectifications analogiques qu'un lecteur humain est en mesure de faire quand une information est incomplète. Ce type de dictionnaire ne présuppose pas une connaissance préalable de la langue et du monde. Seules les informations qui lui sont fournies et sa structuration permettent de reconnaître ou de construire les phrases d'une langue naturelle. Un dictionnaire électronique est une base de données cohérente, qui n'existe que sur support électronique et qui n'a donc pas subi d'informatisation. Il sera directement utilisable par l'ordinateur.

Observons comment est traité linguistiquement un verbe de perception comme voir dans un dictionnaire informatisé monolingue du type Le Robert :

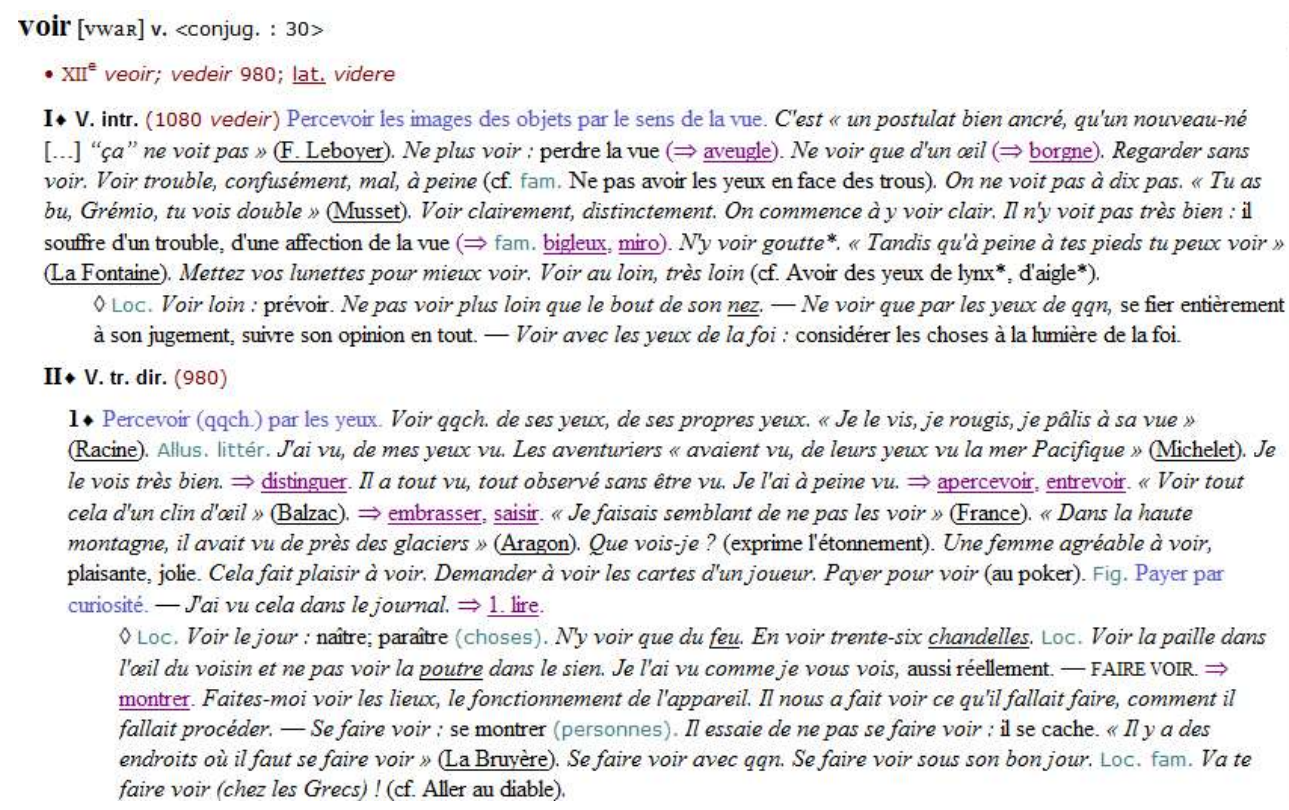

Figure 1 : Extrait de l'entrée voir (CD Rom Le Petit Robert)

Les données telles qu'elles se trouvent dans la définition présentent de nombreux inconvénients et faiblesses (CATACH, 2000). Nous avons un enchevêtrement d'informations de différentes natures (définitions, exemples, expressions, etc.), des illogismes de classification de sens et de synonymie, des renvois hypertextes non ciblés, etc. Si l'on prend simplement l'exemple des locutions au sein de la définition (voir loin, voir le jour, se faire voir avec quelqu'un, etc.), on constate une description lexicographique assez pauvre. Les informations d'emplois de ces locutions sont pratiquement inexistantes. De façon assez surprenante également, on trouve dans le sens 1 de l'emploi transitif direct («percevoir quelque chose par les yeux ») des locutions comme voir le jour ou se faire voir qui sont sémantiquement très éloignées de ce sens.

Nous voyons que les technologies informatiques, dans le cadre des dictionnaires informatisés, si elles ont transformé le mode d'accès aux données contenues dans 
ces documents ${ }^{1}$, ne semblent cependant pas avoir été exploitées au maximum par les lexicographes et les éditeurs. Ces améliorations n'ont qu'un impact limité sur l'exploitation des richesses du dictionnaire dans le cadre de l'apprentissage d'une langue. Ainsi, du point de vue de la description des propriétés linguistiques des unités lexicales, un important travail reste à faire. L'informatisation des dictionnaires papier, bien que constituant une première approche dans le traitement du langage par ordinateur (puisqu'elle génère des outils uniquement consultables sur ordinateur), ne répond pas à une véritable description formelle du langage ${ }^{2}$.

Aux dictionnaires informatisés, mis à la disposition de l'utilisateur humain doté d'une connaissance linguistique, s'opposent les dictionnaires électroniques, les bases de données, uniquement élaborés pour être utilisés par des programmes informatiques... et dépourvus, normalement, de connaissances linguistiques. Face au caractère inachevé des dictionnaires traditionnels et à leur grande part d'implicite apparait la nécessaire complétude des dictionnaires électroniques susceptibles d'analyser les mots d'un texte et le caractère obligatoirement explicite de leurs informations. Enfin, l'association étroite des dictionnaires électroniques à des programmes de traitement automatique impose une cohérence structurelle des entrées. L'émergence de ces dictionnaires s'inscrit dans la mouvance du traitement automatique du langage (TAL), discipline visant à traiter de façon automatique, c'est-à-dire par le biais de l'outil informatique, les données linguistiques de tout genre (COUGNON et FAIRON, 2009).

\section{Morfetik}

Les dictionnaires morphologiques, entre autres, sont utiles pour l'analyse automatique des textes. Dans cette perspective, nous avons élaboré la ressource linguistique Morfetik. Cette ressource est le résultat du travail d'une vingtaine d'années de collecte et de description (MATHIEU-COLAS, 2009 ; GREZKA, CARTIER et MATHIEU-COLAS, 2015) $)^{3}$. Morfetik est un dictionnaire morphologique des mots simples du français. Le système ainsi conçu permet de générer automatiquement l'ensemble des formes simples du français - 758035 formes fléchies pour 103312 lemmes (compte tenu des homographies) - en l'état actuel de la description : 66393 noms, 10223 verbes et 24391 adjectifs (2 305 autres). L'inventaire n'est pas clos. Actuellement, nous recensons l'ensemble des rectifications orthographiques du français de 1990 (trait d'union remplacé par la soudure, accent circonflexe, etc.).

Cette ressource permet d'obtenir, pour n'importe quel mot français, l'ensemble de ses formes (pluriel des noms, féminin et pluriel des adjectifs, formes conjuguées

\footnotetext{
${ }^{1}$ Les versions électroniques des dictionnaires ont simplifié l'accès aux informations contenues dans le dictionnaire par la mise en place de fonctionnalités de recherche plein texte et paramétrables et par les améliorations apportées à la lisibilité de l'article (mise en page, mise en évidence de la structure de l'article, etc.).

2 Dans la pratique didactique courante, le dictionnaire informatisé s'utilise essentiellement comme ouvrage d'appoint dans des situations de communication quotidiennes d'aide à la compréhension ou d'aide à la rédaction.

${ }^{3}$ Travail sous la direction à l'époque de M. Mathieu-Colas. Depuis 2014, nouvelle équipe (CNRS, universitaires, doctorant) dirigée par A. Grezka : J.F. Sablayrolles ; E. Cartier ; C. Jacquet-Pfau ; F. Martin-Berthet; M. Mathieu-Colas ; W. Dekdouk; L. Catach.
} 
des verbes), ou bien, réciproquement, d'identifier le mot (la forme de base, le "lemme") correspondant à n'importe quelle forme fléchie. Un ensemble d'outils de navigation et de consultation de la ressource a ainsi été développé :

- outil de lemmatisation de formes : à partir d'une forme quelconque, l'outil renvoie à l'ensemble des lemmes pouvant y correspondre ;

- outil de génération des flexions : à partir d'une zone permettant de saisir une forme, l'outil fournit les différents lemmes possibles, puis les différentes formes liées à chaque lemme ;

- moteur de recherche sur les formes et/ou les flexions: un moteur de recherche permet de lancer des recherches classiques sur l'ensemble des champs disponibles dans la base. L'idée est de proposer aux chercheurs un moteur de recherche permettant d'effectuer des recherches complexes sur la base lexicographique.

La ressource peut soit être consultée via un moteur de recherche, soit téléchargée dans un format $\mathrm{XML}^{4}$.

\section{Illustration de Morfetik avec les verbes de perception : structuration interne}

Pour notre test et notre démonstration, nous avons utilisé le matériel lexical sur la perception de GREZKA A. (2006), soit :

\begin{tabular}{|l|c|}
\hline \multicolumn{1}{|c|}{ MATERIEL LEXICAL } & $\begin{array}{c}\text { NOMBRE } \\
\text { D'ENTREES }\end{array}$ \\
\hline Verbes de perception & 129 \\
\hline $\begin{array}{l}\text { Verbes d'émission de phénomènes sensibles (visuel, sonore, } \\
\text { olfactif) }\end{array}$ & 424 \\
\hline Adjectifs de sensation & 1142 \\
\hline $\begin{array}{l}\text { Noms relatifs aux phénomènes auditifs, olfactifs et gustatifs (bruit, } \\
\text { odeur, goût) }\end{array}$ & 2903 \\
\hline Noms relatifs aux instruments de perception & 123 \\
\hline
\end{tabular}

En interne (c'est-à-dire la structuration non visible par l'utilisateur), les données de Morfetik ${ }^{5}$ sont structurées sous forme de tables et servent de point de départ à un système de traitement qui combine un moteur de flexion, un dictionnaire des formes fléchies, des interfaces de consultation et d'interrogation, ainsi qu'un ensemble d'outils permettant la maintenance et l'exploitation des ressources. La structure des tables diffère selon les catégories morphosyntaxiques : 5 groupes distincts ont été constitués (adverbes, déterminants/pronoms, noms, adjectifs et verbes).

\footnotetext{
${ }^{4}$ A partir du site du laboratoire LDI : https://outilsldi.univ-paris13.fr/morfetik/

${ }^{5}$ Les données ont été établies à partir de nombreuses sources lexicographiques, notamment : le DELAS (Dictionnaire électronique du LADL, cf. B. CourToIs 1990); le Petit et le Grand Robert; le Petit Larousse illustré, le Lexis, le Grand Larousse encyclopédique et le Grand Dictionnaire encyclopédique Larousse (GDEL) ; le Trésor de la langue française ; le Harrap's et le Robert \& Collins; des dictionnaires d'argot; des tables de conjugaison (dont le Bescherelle et les Verbes logiques de A. Dugas); Le Bon Usage de Grevisse et des dictionnaires de «difficultés » pour le traitement des cas problématiques.
} 
Ainsi, pour les mots invariables, comme les adverbes, un listage est suffisant. En revanche, pour les déterminants et les pronoms, les lemmes sont accompagnés de l'énumération des formes; par exemple, pour un:

\begin{tabular}{|l|l|l|l|l|}
\hline Lemme & CatGram & Forme & Genre & Nombre \\
\hline un & D:Ind & un & M & S \\
\hline un & D:Ind & une & F & S \\
\hline un & D:Ind & des & M & P \\
\hline un & D:Ind & des & F & P \\
\hline un & D:Num & un & & \\
\hline un & P:Ind & un & M & S \\
\hline un & P:Ind & une & F & S \\
\hline un & P:Ind & uns & M & P \\
\hline un & P:Ind & unes & F & P \\
\hline
\end{tabular}

Figure 2 : Extrait de la table des déterminants

Pour les mots les plus complexes (noms, adjectifs et verbes), deux tables complémentaires ont été réalisées :

(i) des tables de flexion permettant d'identifier et de coder tous les types de formation (pluriels, conjugaisons, etc.)

(ii) des tables attribuant à chaque lemme le code flexionnel correspondant (utilisées ensuite par le moteur de flexion pour produire l'ensemble de toutes les formes fléchies).

Ce sont ces tables qui seront ensuite utilisées par le moteur de flexion pour produire l'ensemble de toutes les formes fléchies.

Pour les noms, la table des codes comporte 63 codes. Outre les pluriels courants, toutes les formes particulières, y compris les nombreux types d'emprunts, reçoivent un code spécifique. Pour des raisons à la fois théoriques et pratiques, les noms n'ont pas été fléchis en genre (il n'y a donc dans la nomenclature que des noms masculins et des noms féminins) :

\begin{tabular}{|c|c|c|c|}
\hline Code & Radical & S & P \\
\hline 00 & 0 & & \\
\hline 01 & 0 & & S \\
\hline 02 & 0 & & $\mathrm{x}$ \\
\hline 03 & 1 & I & ux \\
\hline 06 & 4 & œil & yeux \\
\hline $1 D$ & 2 & us & i \\
\hline
\end{tabular}




\begin{tabular}{ll} 
exemple (nm) & exemple (nf) \\
sens; albinos & albinos \\
\hline toucher & vue \\
\hline cheveu & peau \\
$>$ bilatéral & \\
$>$ & oil \\
$>$ & oculus
\end{tabular}

Figure 3 : Extrait des codes flexionnels des noms : les noms de perception

Dans l'extrait, le radical correspond au nombre de caractères à enlever pour construire un radical artificiel utilisé par le fléchisseur pour générer les formes fléchies. Par exemple, pour le mot ceil qui a un pluriel multiple, nous aurons 2 codes :

- le code 01 ( $(x i l / \propto x i l s)$, pour certains emplois pluriels du mot $œ i l$, notamment dans les noms composés (des oils-de-bouf, æils-de-chat, œils-deperdrix, etc.). Ce sont des emplois plus rares de oeils, que l'on va surtout rencontrer dans des domaines spécialisés comme l'armement ${ }^{6}$, l'imprimerie $^{7}$, la marine ${ }^{8}$, ' $^{\text {'outillage }}{ }^{9}$, la technique $;^{10}$

- le code 06 ( d'une plante, etc.

Pour les adjectifs, 59 codes de flexion ont été nécessaires. Contrairement aux noms, les adjectifs sont plus complexes puisqu'ils sont fléchis en genre (en général, une possibilité de quatre formes différentes) :

\begin{tabular}{|c|c|c|c|c|c|}
\hline code & rad & MS & MP & FS & FP \\
\hline 34 & 1 & $\mathrm{x}$ & $\mathrm{x}$ & se & ses \\
\hline 40 & 0 & & $s$ & & s \\
\hline 42 & 0 & & $s$ & e & es \\
\hline 82 & 1 & l & ux & le & les \\
\hline
\end{tabular}

${ }^{6}$ Eils : ouverture ménagée dans un obus pour y introduire la charge d'éclatement et visser la fusée.

${ }^{7}$ Eils : partie du caractère représentant le dessin de la lettre reproduit à l'impression sur le papier pendant le tirage.

${ }^{8}$ Eils : boucle formée à l'extrémité d'un filin.

${ }^{9}$ Eils : trou pratiqué dans la tête d'un marteau pour y fixer le manche.

${ }^{10}$ Eils : judas optique. 


\section{exemples}

$>$ harmonieux ; mélodieux ; silencieux

cacophonique ; rude; insonore

criard ; déplaisant

musical

Figure 4 : Extrait des codes flexionnels des adjectifs : adjectifs de sensation

Enfin, pour les verbes, la table comprend 226 codes de flexion :

\begin{tabular}{|l|l|}
\hline \multicolumn{1}{|c|}{ CHAMPS } & \multicolumn{1}{c|}{ EXEMPLE } \\
\hline Numéro de code & 063 \\
Radical & -3 \\
Radical-modèle & $\mathrm{V}-$ \\
Désinence & - OIR \\
Désinences des formes conjuguées (45 champs) & -ois, -ois, -oit, -oyons, oyez, -oient... \\
Désinences des participes (5 champs) & -oyant, -u, -ue, -us, -ues \\
\hline
\end{tabular}

Figure 5 : Extrait avec le verbe voir

Chacun des codes associés aux lemmes correspond à un type de conjugaison spécifique qui doit être défini avec précision. Les « radicaux » et « désinences » ainsi décrits ne correspondent pas nécessairement au découpage morphologique traditionnel ${ }^{11}$. Afin de faciliter le traitement automatique, le radical est défini comme le plus petit dénominateur commun : par exemple, ici, V-pour VOIR. A la limite, le radical peut être une forme vide (être, avoir, aller). De manière complémentaire, les désinences (-oir, -ois, -oit, -oyons, etc.) s'ajoutent au radical pour construire les formes fléchies.

Ainsi, à partir de cette double représentation, le moteur de flexion peut produire l'ensemble des formes fléchies. Il peut également être exploité pour reconnaitre des formes inconnues. La ressource permet ainsi d'avoir l'ensemble des formes (pluriel des noms, féminin et pluriel des adjectifs, formes conjuguées des verbes), ou bien, réciproquement, d'identifier le mot (la forme de base, le "lemme") correspondant à n'importe quelle forme fléchie.

Par exemple, si nous tapons « vu », l'interface de la ressource nous proposera le tableau suivant, qui montre les différentes analyses possibles de la forme :

${ }^{11}$ Ce découpage morphologique est un découpage interne, donc non visible par l'utilisateur de la ressource. 


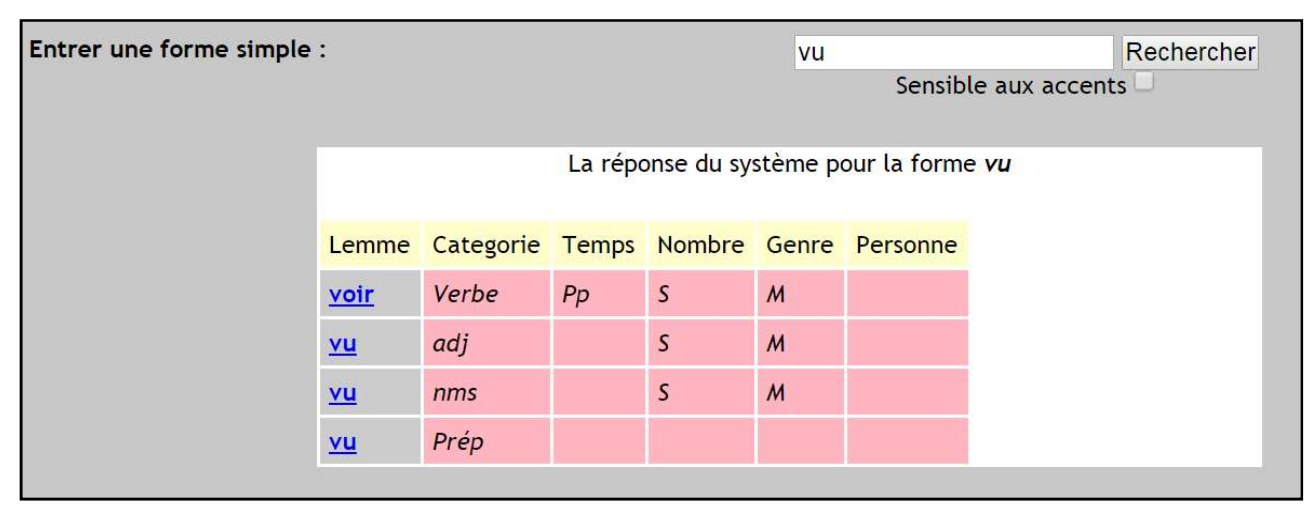

Figure 6 : Résultat d'analyse pour la forme $v u$

L'outil nous renvoie à la fois au lemme, ici à la forme nominale $(v u)$, adjectivale $(v u)$ et verbale (voir), mais également à la préposition $(v u)$. Ensuite, l'utilisateur peut cliquer sur l'un des lemmes pour obtenir les formes correspondantes, et voir ainsi les différentes formes liées à chaque lemme :
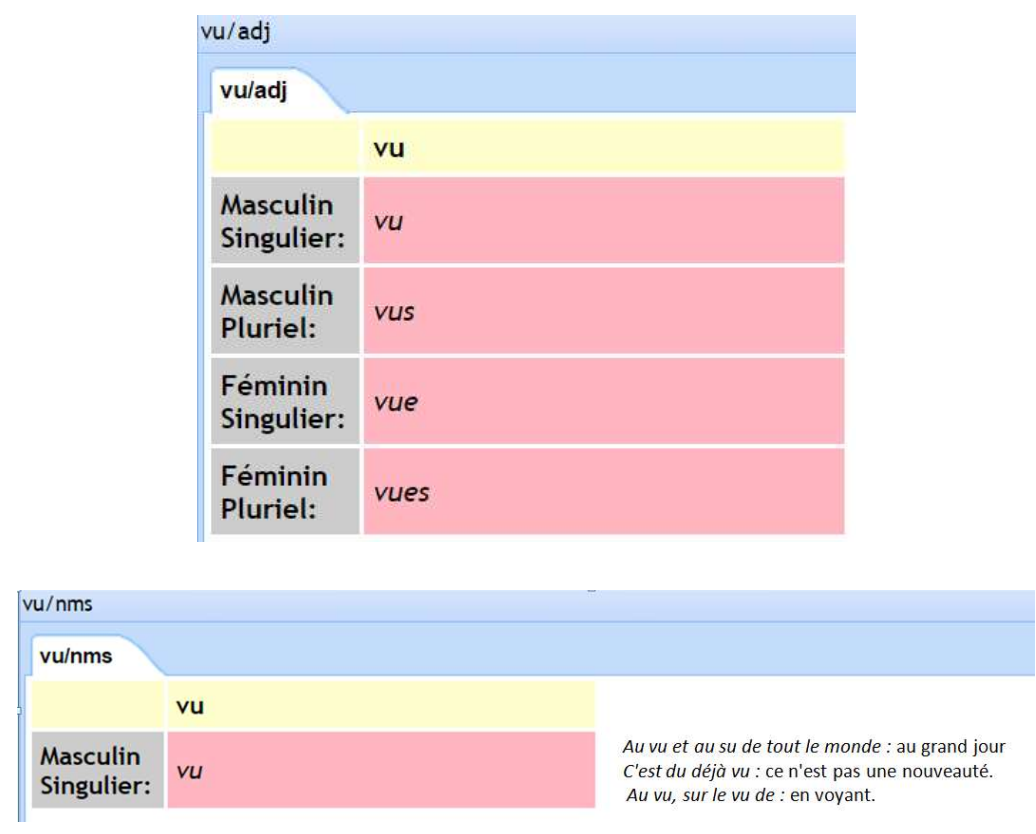


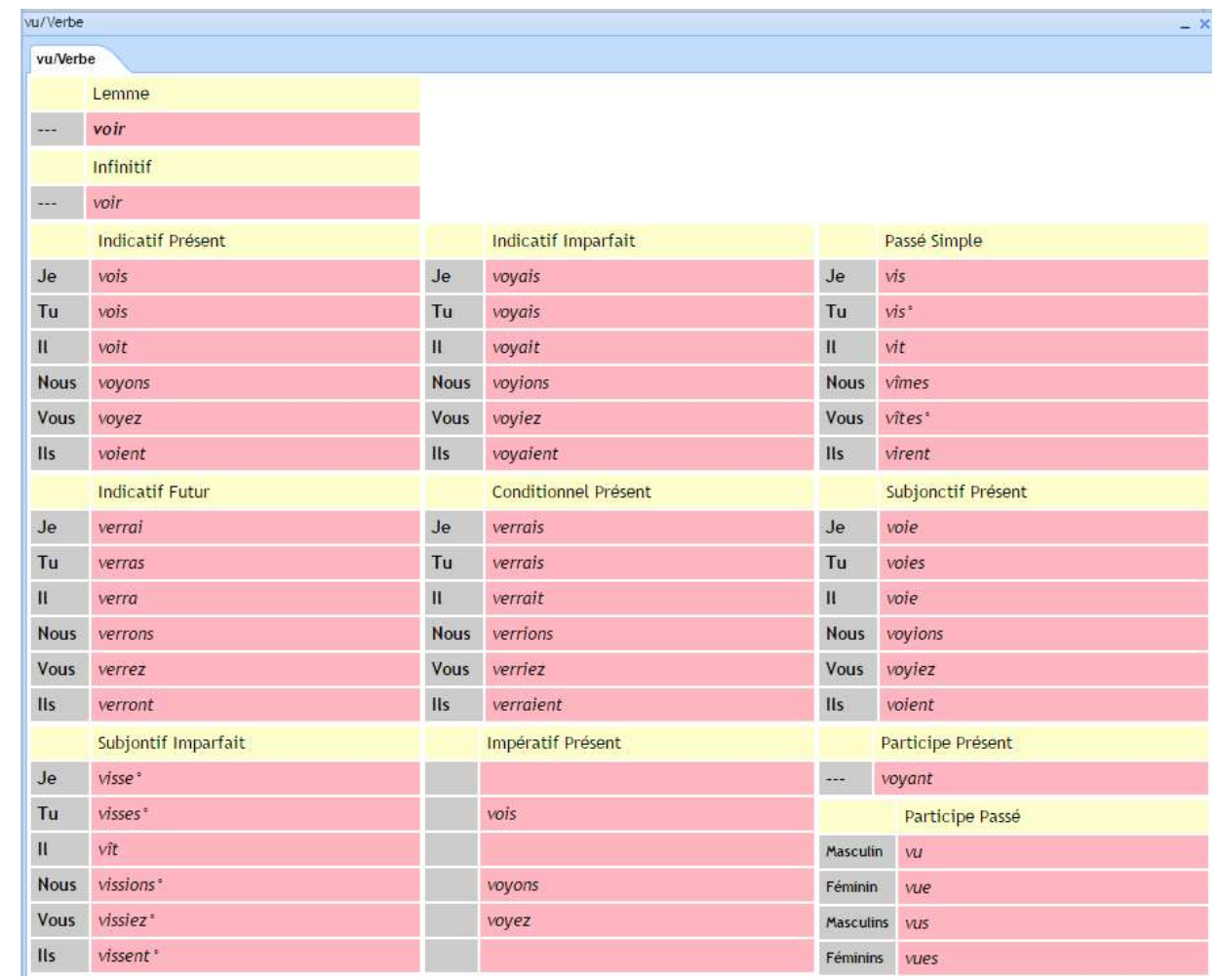

Figure 7 : Résultats de la recherche pour $v u$

Le signe ${ }^{\circ}$ (figure 7) indique les formes rares et/ou archaïques, notamment comme dans cet exemple avec le subjonctif imparfait (visse, visses, vissions, vissiez, vissent). Il permet de formaliser les indications disséminées dans les dictionnaires (le plus souvent, en général, surtout, rarement, etc.). Linguistique, la description permet d'être plus précise et informatiquement, un tel système permet une double utilisation du lexique (standard/exhaustive), selon que les flexions marquées sont ou non prises en compte. Le choix n'est pas négligeable, compte tenu du nombre de formes en jeu. Le marquage peut intervenir à différents niveaux ${ }^{12}$.

À ces données de base viennent s'ajouter des informations complémentaires, permettant la bonne interprétation des données. Par exemple, comme nous pouvons l'observer dans la Figure 7, avec le nom masculin singulier $v u$ qu'on va rencontrer dans des expressions comme au vu et au su de tout le monde; au vu, sur le vu de, etc.

\footnotetext{
12 Sur un plan général, nous marquons :

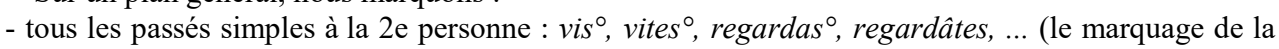
1 re pers. se justifierait moins) ;

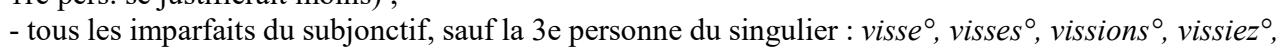
vissent, ...
} 
Les termes spécialisés gagnent également à être marqués comme tels, à l'exemple des noms que nous avons recueillis dans le domaine de l'ophtalmologie :

\begin{tabular}{|c|c|c|c|c|}
\hline ID & Lemme & CatGramm & Flex & Dom \\
\hline N61 & amblyopie & $\mathrm{nf}$ & 01 & med:ophtalm. \\
\hline N80 & astigmatisme & $\mathrm{nm}$ & 01 & med:ophtalm. \\
\hline N233 & blépharite & nf & 01 & med:ophtalm. \\
\hline N523 & cataracte & nf & 01 & med:ophtalm. \\
\hline N575 & cécité & $\mathrm{nf}$ & 01 & med:ophtalm. \\
\hline N1018 & ectropion & $\mathrm{nm}$ & 01 & med:ophtalm. \\
\hline N1019 & entropion & $\mathrm{nm}$ & 01 & med:ophtalm. \\
\hline N1020 & glaucome & $\mathrm{nm}$ & 01 & med:ophtalm. \\
\hline N1021 & hypermétropie & nf & 01 & med:ophtalm. \\
\hline N1023 & kératite & $\mathrm{nf}$ & 01 & med:ophtalm. \\
\hline N1024 & kératocône & $\mathrm{nm}$ & 01 & med:ophtalm. \\
\hline N1125 & névrite optique & $\mathrm{nf}$ & 01 & med:ophtalm. \\
\hline N1227 & uvéite postérieure & $\mathrm{nf}$ & 01 & med:ophtalm. \\
\hline
\end{tabular}

Figure 8 : Résultats de la recherche par domaine, ophtalmologie

\section{Perspectives}

Les systèmes de traitement automatique des langues (TAL) intègrent de plus en plus des connaissances de nature linguistique. Parmi ces connaissances, la morphologie est très souvent utilisée, en particulier dans le traitement des langues comme le français, à morphologie flexionnelle riche mais surtout complexe.

Les dictionnaires morphologiques sont utiles pour l'analyse automatique des textes. Nous avons présenté dans cet article la ressource Morfetik, avec son système de traitement qui associe un moteur de flexion, un dictionnaire des formes fléchies, des interfaces de consultation et d'interrogation, ainsi qu'un ensemble d'outils permettant la maintenance et l'exploitation des ressources.

Cependant, pour être utilisé dans un système de traitement automatique des langues, un dictionnaire de formes n'est jamais suffisant: un correcteur orthographique, un générateur de formes liées notamment à des matrices d'affixation permettant de rendre compte des dérivés sont ainsi inévitables. En effet, de nombreuses études ont montré que l'intégration de la morphologie dérivationnelle à la morphologie flexionnelle améliorait les systèmes de traitement automatique des langues (voir notamment les travaux de JACQUEMIN et al., 1997 ; LEE, 2004). De ce point de vue, une étude supplémentaire doit être menée afin d'utiliser les matrices morphologiques dont dispose Morfetik.

Enfin, ce type de ressources, comme toutes les ressources linguistiques, demande une confrontation continuelle avec des corpus très divers (GREZKA, CARTIER et MATHIEU-COlAS, 2015). Du point de vue des formes linguistiques, cela 
revient à mettre la ressource linguistique en regard avec un corpus continu pour suivre l'évolution fréquentielle des lexies (néologismes, fréquence nulle sur une période, etc.). Une ressource morphologique comme Morfetik est donc l'un des éléments d'un système plus large impliquant un corpus continu et un module de néologismes, ainsi que différents outils pour suivre la fréquence d'usage des lexies du dictionnaire.

\section{BIBLIOGRAPHIE}

CATACH Laurent (2000), Les dictionnaires électroniques des éditions Le Robert, in : DENISE Michel (éd.), Les dictionnaires électroniques, Paris, Didier-CIEP, p. 3142.

COUGNON Louise-Amélie; FAIRON Cédrick (2009), La mise à jour d'un dictionnaire électronique : Une expérience pédagogique liée à la mise à jour du Delaf, Arena Romanistica, 28th Conference on Lexis and Grammar, Bergen (29/09/2009-03/10/2009), vol. 1, no. 4, p. 58-71.

COURTOIS Blandine (1990), Un système de dictionnaires électroniques pour les mots simples du français, in : COURTOIS Blandine, SILBERZTEIN Max (éd.), Dictionnaires électroniques du français, Langue française 87, Paris, Larousse, p. 11-22.

COURTOIS Blandine; SilberzTEIN Max (1990), Dictionnaires électroniques du français, Langue française 87, Paris, Larousse, p. 3-4.

GREZKA Aude (2006), Les prédicats de perception. Traitement de la polysémie. (Les sens des sens). Thèse de doctorat en Sciences du Langage, Université Paris 13, 24 novembre 2006, 834 pages (vol.1 et 2).

GrezKA Aude; CARTIER Emmanuel, MATHIEU-COlas Michel (2015), Dictionnaires morphologiques du français contemporain: présentation de Morfetik, éléments d'un modèle pour le TAL, TALN 2015, Caen, 22-25 juin 2015.

Gross Gaston (2005), Un dictionnaire électronique des adjectifs du français, Cahiers de Lexicologie, Paris, Centre National de la Recherche Scientifique, p. 11-33.

JACQUEMIN Christian; Klavans Judith; TzOUKERMANN Evelyne (1997), Expansion of multi-word terms for indexing and retrieval using morphology and syntax, in: Proceedings of the 35th Annual Meeting of the Association for Computational.

LEE Young-Suk (2004), Morphological Analysis for Statistical Machine Translation, in : Dumais, S. et S. Roukos (éds), Proceedings of HLT- NAACL 2004, 57-60.

MATHIEU-COLAS Michel (2009), Morfetik, une ressource lexicale pour le TAL, Cahiers de Lexicologie, Paris, Centre National de la Recherche Scientifique, p. 137-146. 